\title{
Evaluation of neutrophil-to-lymphocyte ratio and calcitonin concentration for predicting lymph node metastasis and distant metastasis in patients with medullary thyroid cancer
}

\author{
NIZHEN XU, YANBING JIAN, YAXI WANG and WEN TIAN \\ Department of General Surgery, Chinese PLA General Hospital, Beijing 100853, P.R. China
}

Received January 5, 2018; Accepted June 14, 2018

DOI: $10.3892 / \mathrm{mco} .2018 .1727$

\begin{abstract}
The aim of the present study was to investigate potential predictive factors of lymph node metastasis (LNM) and distant metastasis (DM) of medullary thyroid cancer (MTC). A total of 61 patients newly diagnosed with MTC at the General Hospital of the Chinese People's Liberation Army between January 2001 and January 2016 were enrolled and divided into two groups according to the results of preoperative examinations and surgical histopathology as follows: Group NM (without metastases), and group M (with metastases). Univariate logistic regression analysis demonstrated that sex, tumor size, preoperative peripheral blood neutrophil-to-lymphocyte ratio (NLR), and concentration of carcinoembryonic antigen (CEA) and calcitonin (Ctn), were significantly associated with LNM and DM. The multivariate analysis revealed that a Ctn concentration of $>500 \mathrm{pg} / \mathrm{ml}$ [odds ratio $(\mathrm{OR})=21.422 ; 95 \%$ confidence interval $(\mathrm{CI}): 2.611-175.731]$ and the NLR $(\mathrm{OR}=5.918$; 95\% CI: $1.147-30.541)$ were positively correlated with LNM and DM. The optimal cut-off value of the NLR for predicting LNM and DM obtained from receiver operating characteristic curve analysis was 1.784 (sensitivity $68.3 \%$ and specificity $80 \%$ ), and the area under the curve was 0.717 . In conclusion, the findings of the present study strongly suggest that inflammation and immune activation of MTC cells promote LNM and DM, and that higher values of NLR and $\mathrm{Ctn}$ concentration confer a high risk of metastasis.
\end{abstract}

\section{Introduction}

Medullary thyroid carcinoma (MTC) is a rare neuroendocrine tumor, which arises from parafollicular calcitonin-secreting cells (C-cells), and accounts for 3-10\% of thyroid malignancies,

Correspondence to: Professor Wen Tian, Department of General Surgery, Chinese PLA General Hospital, 28 Fuxing Road, Beijing 100853, P.R. China

E-mail: tianwen301_cta01@163.com

Key words: medullary thyroid cancer, lymph node metastasis, distant metastasis, predictive factors, neutrophil-to-lymphocyte ratio, calcitonin although MTC is responsible for up to $13.4 \%$ of thyroid cancer-related deaths (1). MTC is of intermediate malignancy, characterized by partly invasive growth, and is more prone to generating lymph node metastasis (LNM) and distant metastasis (DM) compared with differentiated thyroid cancer, with $\sim 10-15 \%$ of MTC patients having DM when first diagnosed. Unfortunately, the number of available studies on the predictors of metastasis in patients with MTC is limited.

Sex, age, tumor size, calcitonin (Ctn) and carcinoembryonic antigen (CEA) concentrations, among other factors, have been found to be associated with MTC (2-4). Ctn is a 32-amino acid residue peptide, encoded by a cognate gene located on the short arm of chromosome 11 in the C-cell. Ctn serves as a very sensitive and specific tumor marker for the diagnosis of MTC (5).

Neutrophils are the most abundant leukocyte population in the circulation and are the first cells recruited to the site of infection or inflammation (6). Neutrophils are common infiltrating cells in acute inflammation and a component of chronic inflammatory infiltrates. Research suggests that an inflammatory environment contributes to tumor angiogenesis, mutations, cell migration and metastatic progression through this particular environment that promotes the growth of malignant cells (7-9).

Numerous studies have reported that neutrophils play a critical role in tumor progression through the release of cytokines and angiogenic factors (10). Lymphocytes, which are key factors involved in the immune surveillance of tumor cells (11), kill cancer cells to suppress the progression of cancer. Furthermore, the development of several malignant tumors is closely correlated with an imbalance in the size of the populations of functional lymphocytes. A growing body of evidence highlights the role of lymphocytes as a central factor associated with the prognosis of patients with locally advanced cancer (12).

The systemic inflammatory response, a subject of intensive research, suppresses the activity of host immune cells through promoting microvascular regeneration, which facilitates the proliferation and differentiation of tumor cells, thus enhancing the invasion of tissues by tumor cells (13-15). The neutrophil-to-lymphocyte ratio (NLR) serves as an accurate and reliable index of systemic inflammation and, thus, serves as a systemic marker of inflammation (16). Furthermore, strong evidence indicates that a higher NLR is associated with the growth and migration of malignant cells. 
Numerous studies suggest that the initiation and progression of thyroid cancer is closely associated with inflammation (17). Moreover, neutrophils indicate the presence of inflammation, and lymphocytes reflect the function of the immune system. The NLR is a relevant factor of several malignancies, such as urothelial carcinoma (18), lung cancer (19), gastric cancer (20) and colorectal cancer (21). Unfortunately, the number of studies assessing the clinical factors that may help predict those MTC patients who have metastases is limited. The aim of the present study was to investigate the clinical data of 61 patients with MTC to identify predictors of metastasis.

\section{Patients and methods}

Patients. A retrospective analysis of 61 patients newly diagnosed with MTC at the General Hospital of the Chinese People's Liberation Army (PLAGH) between January 2001 and January 2016 was performed. The patients were diagnosed according to the findings of the histopathological examination. The inclusion criteria were as follows: i) MTC was confirmed using thyroid tissue obtained by ultrasound-guided aspiration biopsy or surgery; ii) the patients were not administered chemotherapy, radiotherapy or hormone therapy; iii) routine blood tests were conducted within 3 days prior to surgery or within 1 week prior to biopsy; iv) serum Ctn and CEA assays were conducted within 3 days prior to surgery or within 1 week after diagnosis by biopsy; and v) the patients underwent preoperative ultrasonography of the thyroid gland and lymph nodes, as well as fluorodeoxyglucose (FDG)-positron emission tomography PET/computed tomography (CT). Patients with suspicious foci detected using FDG-PET/CT underwent CT or magnetic resonance imaging (MRI) of the neck, chest and abdomen, as well as emission-CT (ECT) of bone. The exclusion criteria were as follows: i) Infectious disease, ii) other malignancies and iii) inflammatory conditions within 1 month prior to the surgery or biopsy. The final study population comprised 61 patients (27 men and 34 women, aged 25-73 years), 59 of whom had sporadic and 2 hereditary MTC. All the patients in the present study signed written informed consent forms prior to admission, and they acknowledged that their clinical data would be used for clinical studies. The study protocol was granted ethical approval by the Ethics Committee of the General Hospital of the Chinese People's Liberation Army (Beijing, China).

Ultrasound-guided fine-needle aspiration. The fine-needle aspiration procedure was performed using a standard 21-gauge needle. Sampling typically targeted the solid component of the lesion. If there was more than one nodule, a sample was taken from the nodule with suspicious or atypical ultrasound characteristics.

Surgery. When MTC is clinically apparent (thyroid nodule and a positive fine-needle aspiration), measurements of serum Ctn, CEA and calcium concentrations, as well as ultrasound imaging of the neck should be performed. The surgeon should examine the results of thoracic and superior mediastinal CT/MRI scans if enlarged lymph nodes are identified, or if the preoperative serum Ctn concentration is $>400 \mathrm{pg} / \mathrm{ml}$.

The surgical approaches used at our hospital to treat patients with MTC were as follows: i) Total thyroidectomy (TT) with central lymph node dissection for unilateral intrathyroidal tumors sized $\leq 1 \mathrm{~cm}$; preoperative serum Ctn $\leq 400 \mathrm{pg} / \mathrm{ml}$; and absence of suspected central neck or lateral cervical lymph nodes metastases on ultrasound. ii) TT with dissection of the central lymph node compartment (level VI) and dissection of the involved lateral neck compartments (levels II-V) when any of these features are present: Tumor $>1 \mathrm{~cm}$, suspected lateral cervical lymph node metastases on ultrasound, and preoperative serum Ctn concentrations $>400 \mathrm{pg} / \mathrm{ml}$. iii) TT for patients with confined bilateral nodularity, dissection of the central lymph node compartment (level VI), and dissection of the bilateral lateral neck compartments (levels II-V). Patients' sex, age at diagnosis, histological characteristics of the tumors and neck lymph nodes (such as tumor size, focality and extrathyroidal extension) were recorded. All thyroid and lymph node specimens were evaluated by pathologists at the PLAGH.

Blood tests. Routine blood tests were performed within 3 days prior to surgery or within 1 week prior to biopsy. Samples were analyzed using a Sysmex XE-2100 hematology automated analyzer (Sysmex, Kobe, Japan) with the supplied reagents, red blood cell controls and calibrators (Sysmex, Kobe, Japan). The concentrations of serum Ctn and CEA were determined within 3 days prior to surgery or within 1 week after diagnostic biopsy. The analyzer employs a chemiluminescent immunoassay to measure the concentrations of Ctn and CEA. Ctn assessment was performed using an IMMUNITE1000 chemiluminescence analyzer (Siemens AG, Munich, Germany). The detection limits ranged from 2 to $2,000 \mathrm{pg} / \mathrm{ml}$. The optimal Ctn cut-off value was 8.4 and $5.0 \mathrm{pg} / \mathrm{ml}$ for men and women, respectively. CEA measurement was performed using an ARCHITECT i4000 SR chemiluminescence analyzer (Abbott, Chicago, IL, USA). The detection limits of this method range from 0.5 to $1,500 \mathrm{ng} / \mathrm{ml}$. The optimal cut-off value of CEA was $5.0 \mathrm{ng} / \mathrm{ml}$.

Statistical analysis. Statistical analyses were performed using SPSS 21.0 software (IBM, Armonk, NY, USA). The Kolmogorov-Smirnov test was used to ensure that the data were normally distributed, and such data are presented as mean \pm standard deviation, which were subjected to an independent samples t-test to evaluate the significance of differences between groups. Data not normally distributed are presented as median and quartiles, and they were evaluated using the Mann-Whitney test. Data are presented as frequency and rate. Chi-squared tests were used to analyze categorical variables. Receiver operating characteristic (ROC) curves and the area under the ROC curve (AUC) were used to determine the optimal cut-off values for the NLR to predict LNM and $\mathrm{DM}$ in MTC. Then, multivariate logistic regression analyses were used to identify factors independently associated with metastasis. P-values $<0.05$ were considered to indicate statistically significant differences.

\section{Results}

Identification of independent predictors of metastasis. Of the 61 patients with MTC, 41 were confirmed to have metastases (group M), and 20 were confirmed to be metastasis-free (group 
Table I. Single-factor analysis results of lymphatic metastasis and/or distant metastasis of MTC.

\begin{tabular}{|c|c|c|c|c|}
\hline Variables & $\mathrm{N}=61(\%)$ & Group M, n=41 (\%) & Group NM, n=20 (\%) & P-value \\
\hline $\operatorname{Sex}(\%)$ & & & & 0.034 \\
\hline Male & $27(100)$ & $22(81.5)$ & $5(18.5)$ & \\
\hline Female & $34(100)$ & $19(55.9)$ & $15(44.1)$ & \\
\hline Age (years) & & & & 0.689 \\
\hline$<45$ & $28(100)$ & $20(71.4)$ & $8(28.6)$ & \\
\hline$\geq 45$ & $33(100)$ & $22(66.7)$ & $11(33.3)$ & \\
\hline \multicolumn{5}{|l|}{ Maximum nodal axis $(\mathrm{cm})$} \\
\hline$\leq 2$ & $32(100)$ & $18(56.2)$ & $14(43.8)$ & \\
\hline $2-4$ & $21(100)$ & $15(71.4)$ & $6(28.6)$ & 0.055 \\
\hline$>4$ & $8(100)$ & $8(100)$ & $0(0)$ & 0.034 \\
\hline Focality & & & & 0.098 \\
\hline Solitary & $40(100)$ & $24(60)$ & $16(40)$ & \\
\hline Multifocal & $21(100)$ & $17(81)$ & $4(19)$ & \\
\hline \multicolumn{5}{|l|}{$\mathrm{Ctn}(\mathrm{pg} / \mathrm{ml})$} \\
\hline$<20$ & $3(100)$ & $1(33.3)$ & $2(66.7)$ & \\
\hline $20-50$ & $5(100)$ & $1(20)$ & $4(80)$ & 0.2 \\
\hline $50-200$ & $7(100)$ & $2(28.6)$ & $5(71.4)$ & 0.006 \\
\hline $200-500$ & $4(100)$ & $2(50)$ & $2(50)$ & 0.000 \\
\hline$>500$ & $42(100)$ & $35(83.3)$ & $7(16.7)$ & 0.000 \\
\hline $\mathrm{CEA}(\mu \mathrm{g} / \mathrm{l})$, median (range) & $34.3(0.6-731.4)$ & $46.9(4.2-731.4)$ & $16.6(0.66-136.3)$ & 0.007 \\
\hline NLR, Median (range) & $1.8(0.69-5.53)$ & $1.92(0.76-5.53)$ & $1.69(0.69-2.76)$ & 0.019 \\
\hline
\end{tabular}

Significance was set at $\mathrm{P}<0.05$. Group M, MTC with metastasis; group NM, MTC without metastasis; Ctn, calcitonin; CEA, carcinoembryonic antigen; NLR, neutrophil-to-lymphocyte ratio; MTC, medullary thyroid cancer.

Table II. Multivariate logistic analysis.

\begin{tabular}{lcccccc}
\hline Variables & B & SE & Wald $\chi^{2}$ & OR & P-value & $95 \%$ CI \\
\hline Ctn $(>500 \mathrm{pg} / \mathrm{ml})$ & 3.064 & 1.074 & 8.145 & 21.422 & 0.004 & $2.611-175.731$ \\
NLR & 1.778 & 0.837 & 4.509 & 5.918 & 0.034 & $1.147-30.541$ \\
\hline
\end{tabular}

Significance was set at $\mathrm{P}<0.05$. Ctn, calcitonin; NLR, neutrophil-to-lymphocyte ratio; OR, odds ratio; CI, confidence interval.

NM). Single-factor analysis revealed statistically significant differences between groups regarding sex, tumor size, Ctn and CEA concentrations and NLR $(\mathrm{P}<0.05)$. There was no significant difference between the groups according to age and solitary focal/multifocal thyroid carcinoma $(\mathrm{P}>0.05)$ (Table I). Multivariate logistic regression analysis revealed that $\mathrm{Ctn}$ concentrations $>500 \mathrm{pg} / \mathrm{ml}$ and the NLR were independent predictors of metastasis (Table II).

ROC curve analysis and cut-off value of the NLR. ROC curve analysis indicated that the optimum NLR cut-off for lymphatic metastasis and/or distant metastasis was 1.784 (the sensitivity and specificity were 68.3 and $80 \%$, respectively). The AUC value of the NLR was 0.717 (95\% confidence interval: 0.583-0.851) (Fig. 1).

\section{Discussion}

To the best of our knowledge, metastatic MTC is incurable; therefore, the clinician must select patients that are likely to benefit from therapy, balancing the often slow rate of tumor progression associated with good quality of life against the limited efficacy and potential toxicities of local and systemic therapies (22). There are no known predictive factors for LNM and DM of MTC. Ctn produced by thyroid C-cells is the most sensitive diagnostic marker of MTC. The NLR is a simple index of a systemic inflammatory response, and an elevated NLR may reflect an imbalanced inflammatory state that facilitates tumor growth (23).

In the present study, among the 41 patients with metastasis, $40(97.56 \%, 40 / 41)$ had Ctn concentrations $>500 \mathrm{pg} / \mathrm{ml}$ or an 


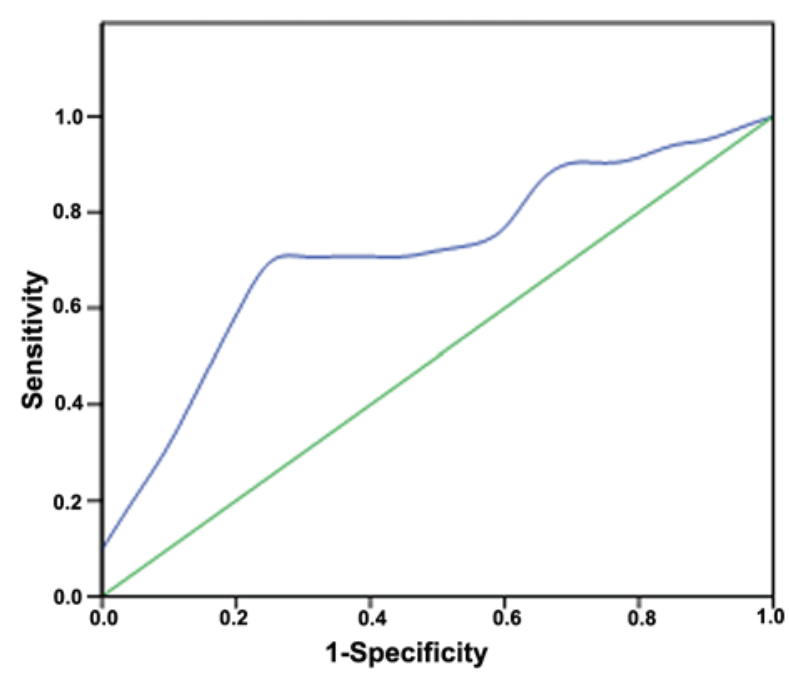

Figure 1. The ROC curve for NLR level of lymphatic metastasis or and/or distant metastasis. The AUC value for NLR is $0.717(\mathrm{P}<0.05)$. NLR, neutrophil-to-lymphocyte ratio; ROC, receiver operating characteristic; AUC, area under the ROC curve.

NLR $>1.784$. Therefore, Ctn concentration and NLR may help predict metastasis. These findings provide compelling evidence that the serum Ctn concentration and the NLR may be used as a primary screen of patients with MTC to detect LNM and DM. Routine implementation of these measurements may facilitate earlier detection of LNM and DM, allowing earlier medical intervention.

MTC is an aggressive tumor, prone to hematogenous and lymphatic metastasis (24). The prognosis of patients with MTC differs between those with differentiated thyroid carcinoma or anaplastic thyroid carcinoma. The tumor metastasizes early to paratracheal and lateral cervical lymph nodes, and distant metastases may develop in the liver, lungs and bones. Given its propensity to spread to lymph nodes and distant organs, MTC is often difficult to cure if not detected early. When lymph node involvement or distant metastases are present at diagnosis, the prognosis is usually poor, even when the primary tumor is quite small (25-27). Therefore, the treatment of such patients differs from that of patients without metastasis, and accurate assessment of a patient's metastatic status is crucial for prescribing optimal individualized treatment that will increase the survival rate.

Ctn, which is produced by thyroid C-cells, is the most sensitive marker available for the diagnosis of MTC. Moreover, the concentration of serum $\mathrm{Ctn}$ represents an accurate and sensitive marker for preoperative diagnosis and post-surgical follow-up. For example, Ctn concentrations $>100 \mathrm{pg} / \mathrm{ml}$ have a positive predictive value (PPV) of $100 \%$ for MTC (27). The PPV associated with Ctn concentrations $\geq 50$ and $<100 \mathrm{pg} / \mathrm{ml}$ vs. Ctn concentrations $\geq 20$ and $<50 \mathrm{pg} / \mathrm{ml}$ is 25 and $8.3 \%$, respectively. The preoperative basal serum Ctn concentration helps assess the extent of lymph node metastasis (22). By contrast, CEA is not a specific biomarker of MTC, although serum CEA concentrations are useful for evaluating disease progression in patients with clinically evident MTC. Furthermore, the preoperative serum CEA concentration is correlated with the number of metastatic lymph nodes, and combining the Ctn and CEA data increases the rate of successful diagnosis.
Kazaure et al (1) advocate that the size of the primary tumor and extrathyroid extension is independently associated with the prognosis of MTC, whereas Dequanter and Lothaire (3) consider age to be an important prognostic factor. Furthermore, the basal mortality rate of MTC reveals that survival is independent of age (28). Inflammation is implicated in the initiation and progression of thyroid cancer. For example, the molecular inflammatory process plays a central role in the malignant progression of transformed thyroid cells $(29,30)$. The NLR is a simple index of the systemic inflammatory response and serves as a prognostic indicator in certain cancers. Liu et al (23) suggested that an elevated NLR may serve as a marker of an imbalanced inflammatory state that facilitates tumor growth. Moreover, the NLR serves an independent prognostic predictor of patients with cancer (31-33).

The present study retrospectively analyzed 61 patients with newly diagnosed MTC. Univariate logistic regression analysis revealed that sex, tumor size, preoperative peripheral blood NLR and serum concentrations of CEA and Ctn were associated with LNM and DM. Multivariate analysis revealed that $\mathrm{Ctn}$ concentrations $>500 \mathrm{pg} / \mathrm{ml}$ and NLR are independent predictors of metastasis. The Ctn concentrations in men were markedly higher compared with those in women, which may be explained by the abundance of thyroid C-cells in men (34). Furthermore, Ctn concentrations are inversely correlated with those of CEA and with tumor size $(35,36)$.

The optimal cut-off value of the NLR for predicting metastasis obtained from ROC analysis was 1.784 (sensitivity $68.3 \%$ and specificity $80 \%$, AUC 0.717 ). Furthermore, patients with an NLR $>1.784$ had a significantly higher rate of LNM and $\mathrm{DM}$ compared with patients with an NLR $\leq 1.784$. Among the 41 patients with metastasis, $40(97.56 \%)$ had Ctn concentrations $>500 \mathrm{pg} / \mathrm{ml}$ or an NLR $>1.784$. Previous studies demonstrated that $\mathrm{Ctn}$ concentrations are associated with tumor load (37). Of the 42 patients with Ctn concentrations $>500 \mathrm{pg} / \mathrm{ml}$ in the present study, 35 had LNM or/and DM. By contrast, other studies reported that high $\mathrm{Ctn}$ concentrations are consistent with LNM and higher TNM stage (38). In the present study, among the 32 patients with NLR $>1.784,28$ had LNM or/and $\mathrm{DM}$, supporting the conclusion that a high NLR is associated with metastasis.

There is a strong association between inflammation and MTC. For example, activated neutrophils may directly and indirectly stimulate tumor growth. Lymphocytes participate in the immune process, construct immune barriers, and kill tumor cells. An elevated NLR may indicate an excessive but ineffective immune response to the tumor load, or serve as a marker of an imbalanced inflammatory state, which facilitates tumor growth.

Whole-body imaging of all patients with MTC is costly and labor-intensive. Serum Ctn assays and NLR determination, which are cost-effective and universally available, may be used as a primary screen for MTC patients to detect LNM and DM. Furthermore, high-risk patients should undergo imaging examinations to facilitate early detection of metastasis, earlier diagnosis, and earlier medical intervention.

There were certain limitations to this study. First, this was a retrospective single-center study. Second, the 
limited sample size of MTC patients did not permit us to perform more intensive analyses. Finally, only the NLR in the prediction of LNM and DM in MTC patients was analyzed, whereas no information was reported on other immunological parameters. A multicenter community-based prospective study, a study with a higher number of cases, and a more sophisticated study including eosinophils, basophils or IgG levels and other immunological parameters, are required in the future in order to investigate a more useful and precise method for predicting LNM and MD in patients with MTC.

In summary, the findings of the present study demonstrated that the serum Ctn concentration and the NLR helped predict the presence of LNM and DM in patients with MTC. Therefore, physicians should pay more attention to patients with Ctn concentrations $>500 \mathrm{pg} / \mathrm{ml}$ or an NLR $>1.784$, and then perform imaging examinations to detect metastases as early as possible. These interventions are critically important for efforts to implement individualized treatment that improves the quality of life and prolongs survival.

\section{Acknowledgements}

Not applicable.

\section{Funding}

This study was funded by Center for Diagnosis and Treatment of Thyroid Diseases in Chinese PLA General Hospital.

\section{Availability of data and materials}

All the data collected and analyzed in the study are available from the corresponding author on reasonable request.

\section{Authors' contributions}

All the authors have read and approved the final version of this manuscript. WT and NX designed the present study. NX, YJ and YW performed the experiments. All authors participated in the writing of the manuscript.

\section{Ethics approval and consent to participate}

The study protocol was granted ethical approval by the Ethics Committee of the General Hospital of the Chinese People's Liberation Army (Beijing, China). All the patients in the present study signed written informed consent forms prior to admission, and they acknowledged that their clinical data would been used for clinical studies.

\section{Patient consent to publication}

Not applicable.

\section{Competing interests}

The authors declare that they have no competing interests to disclose.

\section{References}

1. Kazaure HS, Roman SA and Sosa JA: Medullary thyroid microcarcinoma: A population-level analysis of 310 patients. Cancer 118: 620-627, 2012.

2. Chen H, Sippel RS, O'Dorisio MS, Vinik AI, Lloyd RV and Pacak K; North American Neuroendocrine Tumor Society (NANETS): The North American Neuroendocrine Tumor Society consensus guideline for the diagnosis and management of neuroendocrine tumors: Pheochromocytoma, paraganglioma, and medullary thyroid cancer. Pancreas 39: 775-783, 2010.

3. Dequanter D and Lothaire P: Medullary thyroid cancer: Surgical results and prognostic factors. Rev Med Liege 65: 450-452, 2010 (In French)

4. Skinner MA, Moley JA, Dilley WG, Owzar K, Debenedetti MK and Wells SA Jr: Prophylactic thyroidectomy in multiple endocrine neoplasia type 2A. N Engl J Med 353: 1105-1113, 2005.

5. Lee CR, Lee S, Son H, Ban E, Kang SW, Lee J, Jeong JJ, Nam KH, Chung WY and Park CS: Medullary thyroid carcinoma: a 30-year experience at one institution in Korea. Ann Surg Treat Res 91: 278-287, 2016

6. Sionov RV, Assi S, Gershkovitz M, Sagiv JY, Polyansky L, Mishalian I, Fridlender ZG and Granot Z: Isolation and characterization of neutrophils with anti-tumor properties. J Vis Exp: e52933, 2015.

7. Sionov RV, Fridlender ZG and Granot Z: The multifaceted roles neutrophils play in the tumor microenvironment. Cancer Microenviron 8: 125-158, 2015.

8. Tecchio C and Cassatella MA: Neutrophil-derived cytokines involved in physiological and pathological angiogenesis. Chem Immunol Allergy 99: 123-137, 2014.

9. Jiang K, Lei J, Chen W, Gong Y, Luo H, Li Z, Gong R and Zhu J: Association of the preoperative neutrophil-to-lymphocyte and platelet-to-lymphocyte ratios with lymph node metastasis and recurrence in patients with medullary thyroid carcinoma. Medicine (Baltimore) 95: e5079, 2016.

10. Giraldo NA, Becht E, Vano Y, Sautès-Fridman C and Fridman WH: The immune response in cancer: From immunology to pathology to immunotherapy. Virchows Arch 467: $127-135,2015$.

11. Jiang K, Lei J, Li C, Shu K, Li W, Zhang Y, Li Z, Gong R and Zhu J: Comparison of the prognostic values of selected inflammation based scores in patients with medullary thyroid carcinoma: A pilot study. J Surg Oncol 116: 281-287, 2017.

12. Yu Y, Wang H, Yan A, Wang H, Li X, Liu J and Li W: Pretreatment neutrophil to lymphocyte ratio in determining the prognosis of head and neck cancer: A meta-analysis. BMC Cancer 18: 383, 2018.

13. Brücher BL and Jamall IS: Epistemology of the origin of cancer: A new paradigm. BMC Cancer 14: 331, 2014.

14. Kim S, Miller BJ, Stefanek ME and Miller $\mathrm{AH}$ : Inflammation-induced activation of the indoleamine 2,3-dioxygenase pathway: Relevance to cancer-related fatigue. Cancer 121: 2129-2136, 2015.

15. Hainaut $\mathrm{P}$ and Plymoth A: Targeting the hallmarks of cancer: Towards a rational approach to next-generation cancer therapy. Curr Opin Oncol 25: 50-51, 2013.

16. Liu JF, Ba L, Lv H, Lv D, Du JT, Jing XM, Yang NJ, Wang SX, Li C and Li XX: Association between neutrophil-to-lymphocyte ratio and differentiated thyroid cancer: A meta-analysis. Sci Rep 6: 38551, 2016.

17. Kocer D, Karakukcu C, Karaman H, Gokay F and Bayram F: May the neutrophil/lymphocyte ratio be a predictor in the differentiation of different thyroid disorders? Asian Pac J Cancer Prev 16: 3875-3879, 2015.

18. Taguchi S, Nakagawa T, Matsumoto A, Nagase Y, Kawai T, Tanaka Y, Yoshida K, Yamamoto S, Enomoto Y, Nose Y, et al: Pretreatment neutrophil-to-lymphocyte ratio as an independent predictor of survival in patients with metastatic urothelial carcinoma: A multi-institutional study. Int J Urol 22: 638-643, 2015.

19. Huang C, Yue J, Li Z, Li N, Zhao J and Qi D: Usefulness of the neutrophil-to-lymphocyte ratio in predicting lymph node metastasis in patients with non-small cell lung cancer. Tumour Biol 36: 7581-7589, 2015.

20. Chen J, Hong D, Zhai Y and Shen P: Meta-analysis of associations between neutrophil-to-lymphocyte ratio and prognosis of gastric cancer. T World J Surg Oncol 13: 122, 2015. 
21. Choi WJ, Cleghorn MC, Jiang H, Jackson TD, Okrainec A and Quereshy FA: Preoperative neutrophil-to-lymphocyte ratio is a better prognostic serum biomarker than platelet-to-lymphocyte ratio in patients undergoing resection for nonmetastatic colorectal cancer. Ann Surg Oncol 22 (Suppl 3): S603-S613, 2015.

22. Wells SA Jr, Asa SL, Dralle H, Elisei R, Evans DB, Gagel RF, Lee N, Machens A, Moley JF, Pacini F, et al: Revised American Thyroid Association guidelines for the management of medullary thyroid carcinoma. Thyroid 25: 567-610, 2015.

23. Liu CL, Lee JJ, Liu TP, Chang YC, Hsu YC and Cheng SP: Blood neutrophil-to-lymphocyte ratio correlates with tumor size in patients with differentiated thyroid cancer. J Surg Oncol 107: 493-497, 2013

24. Hazard JB, Hawk WA and Crile G Jr: Medullary (solid) carcinoma of the thyroid; a clinicopathologic entity. J Clin Endocrinol Metab 19: 152-161, 1959.

25. Yamazaki M, Straus FH, Messina M, Robinson BG, Takeda T, Hashizume $\mathrm{K}$ and DeGroot LJ: Adenovirus-mediated tumor-specific combined gene therapy using Herpes simplex virus thymidine/ganciclovir system and murine interleukin-12 induces effective antitumor activity against medullary thyroid carcinoma. Cancer Gene Ther 11: 8-15, 2004.

26. Hyer SL, Vini L, A'Hern R and Harmer C: Medullary thyroid cancer: Multivariate analysis of prognostic factors influencing survival. Eur J Surg Oncol 26: 686-690, 2000.

27. Costante G, Meringolo D, Durante C, Bianchi D, Nocera M, Tumino S, Crocetti U, Attard M, Maranghi M, Torlontano M and Filetti S: Predictive value of serum calcitonin levels for preoperative diagnosis of medullary thyroid carcinoma in a cohort of 5817 consecutive patients with thyroid nodules. J Clin Endocrinol Metab 92: 450-455, 2007.

28. de Groot JW, Plukker JT, Wolffenbuttel BH, Wiggers T, Sluiter WJ and Links TP: Determinants of life expectancy in medullary thyroid cancer: Age does not matter. Clin Endocrinol (Oxf) 65: 729-736, 2006

29. De Santis E, Di Vito M, Perrone GA, Mari E, Osti M, De Antoni E, Coppola L, Tafani M, Carpi A and Russo MA: Overexpression of pro-inflammatory genes and down-regulation of SOCS-1 in human PTC and in hypoxic BCPAP cells. Biomed Pharmacother 67: 7-16, 2013.
30. Guarino V, Castellone MD, Avilla E and Melillo RM: Thyroid cancer and inflammation. Mol Cell Endocrinol 321: 94-102, 2010.

31. Malietzis G, Giacometti M, Askari A, Nachiappan S, Kennedy RH, Faiz OD, Aziz O and Jenkins JT: A preoperative neutrophil to lymphocyte ratio of 3 predicts disease-free survival after curative elective colorectal cancer surgery. Ann Surg 260: 287-292, 2014.

32. Feng JF, Huang Y, Zhao Q and Chen QX: Clinical significance of preoperative neutrophil lymphocyte ratio versus platelet lymphocyte ratio in patients with small cell carcinoma of the esophagus. ScientificWorldJournal 2013: 504365, 2013.

33. Lee S, Oh SY, Kim SH, Lee JH, Kim MC, Kim KH and Kim HJ: Prognostic significance of neutrophil lymphocyte ratio and platelet lymphocyte ratio in advanced gastric cancer patients treated with FOLFOX chemotherapy. BMC Cancer 13: 350, 2013.

34. Basuyau JP, Mallet E, Leroy M and Brunelle P: Reference intervals for serum calcitonin in men, women, and children. Clin Chem 50: 1828-1830, 2004.

35. Boschin IM, Torresan F, Toniato A, Zane M, Ide EC, Pennelli G, Rampin L, Colletti PM, Rubello D and Pelizzo MR: Incidental medullary thyroid microcarcinoma revealed by mild increase of preoperative serum calcitonin levels: Therapeutic implications. Endocrine 45: 448-453, 2014.

36. Saltiki K, Rentziou G, Stamatelopoulos K, Georgiopoulos G, Stavrianos C, Lambrinoudaki E and Alevizaki M: Small medullary thyroid carcinoma: Post-operative calcitonin rather than tumour size predicts disease persistence and progression. Eur J Endocrinol 171: 117-126, 2014

37. Kihara M, Miyauchi A, Kudo T, Hirokawa M and Miya A: Reference values of serum calcitonin with calcium stimulation tests by electrochemiluminescence immunoassay before/after total thyroidectomy in Japanese patients with thyroid diseases other than medullary thyroid carcinoma. Endocr J 63: 627-632, 2016.

38. Romero-Lluch AR, Cuenca-Cuenca JI, Guerrero-Vázquez R, Martínez-Ortega AJ, Tirado-Hospital JL, Borrego-Dorado I and Navarro-González E: Diagnostic utility of PET/CT with 18F-DOPA and 18F-FDG in persistent or recurrent medullary thyroid carcinoma: The importance of calcitonin and carcinoembryonic antigen cutoff. Eur J Nucl Med Mol Imaging 44: 2004-2013, 2017. 